\title{
Fatores de risco para o desenvolvimento de crianças atendidas em Unidades de Saúde da Família, ao final do primeiro ano de vida: aspectos sociodemográficos e de saúde mental materna
}

\author{
Risk factors for impaired development in children \\ attended at family health units at the end of the first year of life: \\ socio-demographic aspects and maternal mental health
}

Débora Gerardo Ribeiro ${ }^{1}$

Gimol Benzaquen Perosa ${ }^{1}$

Flávia Helena Pereira Padovani ${ }^{1}$

${ }^{1}$ Departamento de Neurologia, Psicologia e Psiquiatria, Faculdade de Medicina de Botucatu, Unesp. Caixa Postal 540, Distrito de Rubião Junior. 18.618-970 Botucatu SP Brasil.

deboragerardo@gmail.com
Abstract The scope of this article is to evaluate risk and protection factors for the development of 1-year-olds assisted at family health care units. It is a cross-sectional study involving 65 children of approximately 1 year of age and their mothers attended at two family health care units. The development was assessed using a developmental screening test (Denver II). The mothers filled out the SRQ-20 questionnaire to identify common mental disorder (CMD) indicators. After data collection, descriptive and inferential statistical analysis was performed. Global development was at risk in $43.1 \%$ of the children evaluated, and the most affected areas were language and fine motor development; $44.6 \%$ of mothers had results indicative of CMD when the child was 1 year of age. In bivariate analysis, reported depression, smoking, infections in pregnancy, $C M D$ after birth and working outside the home were significantly associated with the children's development. After full statistical analysis, CMD was revealed as being a risk factor, and working away from home as being a protection factor. In order to increase the chances of success of programs targeted for children at health care units and avoiding the risk of impaired development, it is important to focus on two aspects: children's stimulation and maternal mental health.

Key words Child development, Risk factors, Maternal mental health
Resumo O objetivo deste artigo é verificar fatores de risco e proteção para o desenvolvimento de crianças de 1 ano, atendidas em unidades de saúde da família. Estudo transversal com 65 crianças de aproximadamente 1 ano, atendidas em duas Unidades de Saúde da Família, e suas respectivas mães. Avaliou-se o desenvolvimento a través de um teste de screening para rastreamento de risco. As mães responderam a uma entrevista e ao SRQ-20 para identificar indicadores de transtorno mental comum (TMC). Realizou-se análise descritiva dos dados e procedeu-se a análise estatística inferencial. Estavam em risco para o desenvolvimento global $43,1 \%$ das crianças e as áreas mais afetadas foram linguagem e motricidade fina; $44,6 \%$ das mães pontuaram para indicativo de transtorno mental comum, quando a criança tinha 1 ano. Na análise bivariada, depressão referida, tabagismo, infecções na gravidez, TMC após o nascimento e trabalhar fora associaram-se significativamente com o desenvolvimento da criança. Após os ajustes, apenas TMC apresentou-se como fator de risco e trabalhar fora fator de proteção. Para aumentar as chances de sucesso de programas direcionados a crianças com risco para o desenvolvimento em unidades de saúde parece importante ter dois focos: a estimulação da criança e a saúde mental materna.

Palavras-chave Desenvolvimento infantil, $\mathrm{Fa}$ tores de risco, Saúde mental materna 


\section{Introdução}

Com o objetivo de prevenir doenças e promover o crescimento e o desenvolvimento da criança, uma das prioridades do atual modelo de saúde são o cuidado e a atenção integral à criança, com um acompanhamento longitudinal. Um diagnóstico precoce dos riscos ao desenvolvimento pode aumentar as chances das crianças com atraso, possibilitar um acesso e atenção adequada, proporcionando uma melhor qualidade de vida ${ }^{1}$. Há evidências suficientes de que quanto mais precoces forem o diagnóstico de atraso e a intervenção, menor será o impacto desses problemas no desenvolvimento e na vida futura da criança ${ }^{2}$.

O desenvolvimento tem um caráter multifatorial, é um produto de efeitos diretos e indiretos, complexa combinação de influências que, em face à adversidade, podem ter diferentes desfechos e remetem à necessidade de abordar os fatores e mecanismos de risco e proteção ${ }^{3}$.

São considerados riscos biológicos, acontecimentos pré, peri e pós-natais como prematuridade, baixo peso ao nascimento, complicações no parto e na gravidez. Já entre os fatores de risco ambiental, a literatura refere o baixo nível socioeconômico, a escolaridade e a fragilidade nos vínculos familiares ${ }^{4}$.

Muitas vezes, há concomitância dos dois tipos de risco, biológico e ambiental, provocando um efeito cumulativo. No estudo de Halpern et $\mathrm{al.}^{2}$ a maior chance de atraso no desenvolvimento das crianças esteve associada à parcela da população mais desfavorecida que, por sua vez, acumulava vários fatores de risco biológico: eram pobres, tinham mais de três irmãos, haviam nascido com baixo peso, idade gestacional menor de 37 semanas, e haviam recebido leite materno por menos de três meses ou não haviam sido amamentadas.

O ambiente doméstico tem sido apontado como fundamental quanto à oferta de estímulos que podem influenciar o desenvolvimento infantil. Em um estudo realizado em comunidade de baixa renda na periferia de São Luis (MA), foi analisada a possível associação entre a oferta de estímulos no ambiente familiar e o desenvolvimento de crianças de 2 anos de idade. Os autores concluíram que a qualidade e a quantidade de estímulos ambientais presentes no contexto familiar mostraram-se fundamentais para o desenvolvimento global das crianças ${ }^{5}$. O baixo nível socioeconômico e a pouca escolaridade dos pais podem afetar o desenvolvimento na medida em que se observou uma relação direta entre es- sas variáveis, a responsividade e a qualidade da estimulação materna oferecida à criança. Lordelo et al. ${ }^{6}$ identificaram que os níveis de responsividade de mães cujos filhos tinham de 1 a 3 anos de idade, residentes em bairros de classe média e escolaridade superior eram muito mais altos que daquelas que moravam em bairros de ocupação irregular ("invasão") e tinham pouca escolaridade. Andrade et al. ${ }^{7}$ observaram que o desenvolvimento cognitivo de crianças, entre 17 e 42 meses, moradores de áreas centrais e periféricas de Salvador, esteve associado à qualidade do estímulo doméstico que, por usa vez, estava relacionado com o nível de escolaridade materna.

Outras experiências adversas ligadas ao ambiente familiar como conflitos, doença crônica, problemas de saúde mental do cuidador, podem, também, ser consideradas fatores de risco ${ }^{8}$. Em estudo longitudinal, Laucht et al..$^{9}$ observaram que crianças de 2, 4, 6 e 8 anos, com pais com problemas psiquiátricos, baixo nível educacional, paternidade precoce, conflitos conjugais e várias pessoas vivendo na mesma casa, corriam maior risco de atrasos no desenvolvimento e má adaptação comportamental que crianças nascidas com riscos biológicos.

Para vários autores, a saúde mental materna, no período pré e pós-gestacional, pode ser considerada um fator de risco para o desenvolvimento, na medida em que afeta a qualidade e a disponibilidade do cuidado e a interação mãe/criança e consequentemente, o desenvolvimento infanti ${ }^{10}$.

Há evidencias de que a depressão pré-natal tem forte correlação com temperamento difícil da criança aos 6 meses, atraso no desenvolvimento aos 18 meses, problemas emocionais comportamentais e de atenção em crianças mais velhas. A depressão pré-natal também mostrouse forte preditor da depressão pós-parto e mais comum que esta última ${ }^{11}$. Quanto à depressão pós-parto (DPP), cujos sintomas têm início nas quatro primeiras semanas do pós-parto, devido a sua alta incidência e riscos ao desenvolvimento infantil, é considerada, em alguns contextos, um problema de saúde pública ${ }^{12}$.

Segundo a literatura, a depressão materna após o parto quando não tratada, esteve associada a prejuízos na interação mãe-bebê $\hat{e}^{10,13}$, a alterações eletroencefalográficas ${ }^{14}$, a déficit cognitivo da crianç ${ }^{14,15}$ e a prejuízos emocionais e padrões desajustados de comportamento ${ }^{15}$. A médio e longo prazo, Motta et al. ${ }^{14}$, em artigo de revisão, observaram que filhos de mães que apresentaram depressão pós-parto, aos 12 meses, apresentavam baixo desempenho em testes de 
desenvolvimento e altos níveis de apego inseguro com a mãe.

Apesar de não ser considerada DPP, a manifestação de sintomas depressivos maternos ao longo dos primeiros anos de vida da criança, também podem repercutir negativamente no desenvolvimento ${ }^{12,13}$. Schwengber e Picinini ${ }^{13}$, ao comparar mães com e sem depressão, ao fim do primeiro ano de vida da criança, observaram que as com depressão relatavam mais insatisfação com o desenvolvimento do bebê e desempenho de seu papel, reclamavam da falta de apoio e apresentavam maior estresse no manejo com a criança.

Em comparação com a depressão, a ansiedade materna foi pouco estudada. A ansiedade materna teve efeito negativo sobre a galactopoese e sobre a qualidade do vínculo mãe-bebê, justificando a adoção de medidas preventivas e terapêuticas ${ }^{16}$. Estudo de revisão recente, com levantamento de pesquisas a partir de 2008, só encontrou 3 artigos que associavam ansiedade pós-natal e desenvolvimento, com resultados ainda inconclusivos. Apesar de alguma evidencia de que a ansiedade afetava negativamente o desenvolvimento social e cognitivo, não foi possível comparar os resultados visto que as pesquisas utilizaram diferentes instrumentos de medida, aplicados a crianças de idades muito diversas ${ }^{17}$. A médio e longo prazo, Clavarino et al. ${ }^{18}$ observaram que crianças, cujas mães haviam apresentado altos níveis de ansiedade no pós-parto, aos 5 anos apresentavam desenvolvimento negativo do afeto, e aumento do nível de desatenção.

São vários os fatores que, segundo a literatura, influenciam a saúde mental materna. As condições adversas do meio, assim como historia anterior de depressão, se associaram com aparecimento de quadros depressivos maternos, muitas vezes na fase gestacional. Dificuldades financeiras, baixa escolaridade, desemprego, ausência de suporte social, relações maritais conflituosas, dependência de substancias, violência doméstica e ambivalência com relação ao feto são fortes fatores de risco para depressão durante a gravidez e no pós-parto ${ }^{19}$. Especificamente quanto à ansiedade, mulheres com alta ansiedade-traço, baixa escolaridade, história de humor deprimido apresentaram risco de manifestar ansiedade no puerpério ${ }^{20}$.

Nas últimas décadas, o estado psicológico da mãe, os quadros de depressão ou ansiedade, tem sido foco de preocupação dos pesquisadores e dos serviços de saúde pelo efeito que pode provocar no desenvolvimento infantil, mesmo quando há remissão da patologia materna ${ }^{10,19}$. No entanto, muito pouco se sabe sobre o papel dos chamados transtornos mentais comuns para o desenvolvimento da criança.

Os transtornos mentais comuns, de elevada prevalência na população adulta, especialmente entre as mulheres com baixa condição socioeconômica ${ }^{21,22}$, se caracterizam por múltiplos sintomas como queixas somáticas inespecíficas, irritabilidade, insônia, nervosismo, dores de cabeça, fadiga, esquecimento, falta de concentração. Englobam tanto os indivíduos que apresentam um transtorno psiquiátrico clássico, depressivo, ansioso e somatoforme, classificáveis nos manuais diagnósticos, como aqueles que necessitam de cuidado, mas que não necessariamente satisfazem todos os critérios diagnósticos de doença mental de acordo com as classificações do DSMIV e do CID-10 $0^{23,24}$. A grande maioria desses quadros é subdiagnosticada, podendo não receber o tratamento adequado ${ }^{24}$.

Considerando a relevância da saúde mental materna para a compreensão do desenvolvimento infantil, da pouca literatura a respeito dos transtornos mentais comuns, o presente estudo teve por objetivo verificar fatores de risco e proteção (sociodemográficos e de saúde mental materna) para o desenvolvimento de crianças de 1 ano, atendidas em unidades de saúde. A hipótese principal do estudo foi que menores escores na avaliação do desenvolvimento infantil estariam relacionados à maior frequência de indicadores de transtornos mentais maternos.

\section{Metodologia}

Estudo de corte transversal, realizado durante um período de 11 meses, com crianças e suas respectivas mães, residentes na área de abrangência de duas unidades de saúde da família, situadas em regiões periféricas de um município de médio porte, com 120.000 habitantes, localizado na região centro-oeste do estado de São Paulo.

\section{Participantes}

As crianças foram selecionadas a partir de consulta às planilhas dos Agentes Comunitários de Saúde (ACS) das Unidades de Saúde da Família, e foram convocadas todas que atendessem ao critério de inclusão: crianças com idade variando de 11 meses e 20 dias a 12 meses e 20 dias, durante o período de coleta. Foram estabelecidos como critérios de exclusão: riscos biológicos 
como deficiência mental, motora e sensorial; prematuridade; intercorrências neonatais graves; e longo período de internação após nascimento.

No total, foram convocadas 129 díades. $\mathrm{Na}$ Unidade de Saúde I, do total de 74 crianças, 38 $(51,8 \%)$ concordaram em participar da pesquisa, $16,2 \%$ mudaram da área de abrangência e $35 \%$ recusaram-se a participar. Não houve nenhuma exclusão.

Na Unidade de Saúde II, do total de 55 crianças, $27(49,1 \%)$ participaram da pesquisa, $27,2 \%$ mudaram da área de abrangência e 21,8\% recusaram-se a participar na pesquisa, havendo apenas uma exclusão devido à criança ter grave atraso no desenvolvimento em consequência de doença não identificada no primeiro contato. A amostra final foi constituída por 65 sujeitos, correspondendo à soma dos participantes das duas unidades.

\section{Instrumentos e Materiais}

a) Teste de Triagem do Desenvolvimento de Denver II ${ }^{25,26}$. O instrumento Denver II consiste em um teste de screening para rastreamento de risco, que avalia o desenvolvimento de crianças de um mês a seis anos, em quatro áreas: Pessoal - Social: aspectos da socialização da criança dentro e fora do ambiente familiar; Linguagem: produção de som, capacidade de reconhecer, entender e usar linguagem; Motricidade fina: coordenação olho-mão, manipulação de pequenos objetos; Motricidade ampla: controle motor corporal, sentar, caminhar, pular e todos os demais movimentos realizados pela musculatura ampla.

Os itens são avaliados pelo aplicador diretamente com a criança e, em alguns casos, quando é difícil observar o comportamento no contexto de avaliação, é solicitado que a mãe informe se a criança realiza ou não determinada tarefa. Há cinco possibilidades de registro na folha proposta pelo teste: "P" (passou), "F" (falhou), "NO" (Não houve oportunidade), "RE" (recusa) e "An" se for apenas relato do cuidador.

Para a avaliação final, considera-se "Cuidado" quando a criança falha ou recusa-se a fazer itens em que entre 75 a $90 \%$ da amostra de comparação apresentam tal comportamento (indicado na folha de registro) e "Atraso" quando a criança falha ou recusa-se a fazer itens em que mais de $90 \%$ das crianças da mesma idade já apresentam tal comportamento.
A interpretação final do teste apresenta os indicadores: Normal, quando não houver nenhum "atraso" e, no máximo, um "cuidado"; Risco, quando houver dois ou mais "cuidados" e/ou um ou mais "atrasos" e Não testável se houver marcações de "recusa-se" em um ou mais itens que já deveriam fazer parte do repertório da criança.

b) Roteiro de Entrevista para Identificação de risco e Recursos Biopsicossociais na História de Vida da Criança ${ }^{27}$. O roteiro visa identificar, a partir da história de vida da criança, da mãe e da família a determinação de possíveis riscos de atraso e recursos protetores para o desenvolvimento biológico, psicológico e social. Segundo as autoras, ele foi elaborado a partir de outros utilizados em diferentes estudos. No presente estudo, o instrumento sofreu adaptações devido à etapa de desenvolvimento das crianças incluídas nesta pesquisa. Com as perguntas pretendia-se obter dados sobre os seguintes aspectos:

. socioeconômicos: renda familiar, ocupação, escolaridade dos pais;

. reprodutivos: idade materna, via de parto, assistência pré-natal;

. ambientais/sociais: moradia, saneamento, estado civil da mãe, rede de apoio;

. condições de nascimento da criança: peso ao nascer, idade gestacional, morbidade neonatal;

. atenção à criança: aleitamento materno, vacinação, nutrição, sono, cuidados paternos, creche.

c) Self Report Questionaire-20/SRQ ${ }^{28}$. Tratase de um questionário de rastreamento de distúrbios psiquiátricos em nível de atenção primária em saúde, desenvolvido pela Organização Mundial de Saúde (OMS). É composto por 20 questões com respostas dicotômicas (sim ou não), classificadas em grupos de sintomas físicos e grupos de distúrbios psicoemocionais (diminuição de energia, humor depressivo e pensamento depressivo), para detecção de transtornos mentais comuns, de base neurótica. O próprio instrumento estabelece que a nota de corte, para determinar a possibilidade de transtorno mental comum, é oito. Devem-se pontuar oito ou mais respostas afirmativas para considerar a ocorrência de transtorno mental comum. Suas qualidades psicométricas são consideradas adequadas: sensibilidade entre $63 \%$ e $90 \%$ e especificidade entre $44 \%$ e $95 \%$, tendo sido validado no Brasil $O$ índice de consistência interna (alpha de Cronbach) da versão brasileira foi 0.86. É um instrumento amplamente utilizado em estudos nacionais ${ }^{22,29}$. 


\section{Procedimento}

\section{Coleta de dados}

O presente estudo contou para sua realização com a aprovação do Comitê de Ética em Pesquisa do Hospital das Clínicas da Faculdade de Medicina de Botucatu, da Secretaria Municipal de Saúde do Município, assim como dos responsáveis pelas respectivas USF.

Após a seleção prévia das crianças, as mães eram convidadas a participar da pesquisa, e, caso aceitassem assinavam o Termo de Consentimento Livre e Esclarecido. Eram encaminhadas a uma sala que preservava a privacidade da díade, onde a pesquisadora aplicava, individualmente, os instrumentos às mães, com a criança presente, na seguinte ordem: Self Report Questionaire - 20 e Roteiro de Entrevista para Identificação de Risco e Recursos Biopsicossociais na História de Vida da Criança. No mesmo local, numa segunda etapa, era avaliado o desenvolvimento neuropsicomotor da criança, pelo teste DENVER II.

Todos os instrumentos foram aplicados em uma única sessão, com duração aproximada de 60 minutos.

\section{Análise de dados}

Em um primeiro momento, realizou-se a análise descritiva dos dados, baseada no cálculo de frequência, porcentagem, incidência ou mediana, de acordo com a natureza deles. A seguir, procedeu-se a análise estatística inferencial e considerando que as variáveis analisadas no presente estudo eram categóricas, optou-se pela utilização do teste Qui-quadrado ou teste Exato de Fisher. As variáveis que apresentaram diferenças estatísticas significativas foram incluídas no modelo de análise multivariada, realizada através da análise de regressão logística com o objetivo de identificar o melhor modelo de predição para o desenvolvimento neuropsicomotor global da criança.

O programa usado para as análises foi o Statistical Package for the Social Sciences for Windows 15.0. Os resultados foram discutidos no nível de 5\% de significância.

\section{Resultados}

Das 65 crianças estudadas 50,7\% eram do sexo feminino e apenas 3\% frequentavam a creche. Todas as crianças nasceram a termo, 80,0\% de parto normal e $92,3 \%$ delas tiveram alta junto com a mãe. Foram amamentadas no peito $89,2 \%$ e a mediana do tempo de desmame foi de 8,5 meses. Estavam com a vacinação em dia 97\%. Das mães, $89,3 \%$ referiram que os filhos tinham sono normal e alimentavam-se bem (77\%), mas $66,1 \%$ foram descritos de forma negativa, como crianças "nervosas", "agitadas” ou "birrentas". A maioria das crianças adquiriu as habilidades como sustentar a cabeça, sentar, engatinhar, no tempo adequado, e $40 \%$ já andava.

A maioria dos pais eram adultos jovens: $47,8 \%$ das mães e $60,0 \%$ dos pais tinham entre 20 e 30 anos. Com relação à escolaridade, 55,3\% das mães e $63,0 \%$ dos pais tinham 8 ou mais anos de estudo. Se a grande maioria das mães era dona de casa $(80,0 \%), 95,3 \%$ dos pais estavam empregados e 36,9\% exerciam uma ocupação qualificada.

Viviam em união consensual $87,0 \%$ das famílias, em casa própria $(44,6 \%)$, com água encanada $(98,4 \%)$, rede de esgoto $(78,5 \%)$, localizadas em ruas asfaltadas $(70,6 \%)$. A mediana era de 5 cômodos por casa, com uma variação de 3 a 8. Das famílias, $35 \%$ dividiam a casa com outros familiares: avós, tios e primos das crianças. A mediana da renda da família, em salários mínimos, foi de 1,64 com valor mínimo 0,21 e valor máximo de 4,3 salários.

Com relação à saúde materna, todas as mães relataram ter feito o acompanhamento pré-natal em suas respectivas unidades de saúde. Apresentaram infecção urinária durante a gestação $46,2 \%$ e 30\% eram tabagistas. Quanto à saúde mental 17,0\% das mães relataram depressão na gestação e na avaliação pelo SRQ-20, 44,6\% delas pontuaram para indicativo de transtorno mental comum.

Com relação à avaliação do desenvolvimento, $43,1 \%$ das crianças avaliadas estavam em risco para o desenvolvimento global, sendo que as áreas mais afetadas foram a linguagem $(24,6 \%)$ e o desenvolvimento motor fino $(20,0 \%)$. Uma porcentagem pequena de crianças $(6,1 \%)$ apresentou atraso no domínio pessoal-social, enquanto que o desenvolvimento motor grosso apresentou $100 \%$ de normalidade (Tabela 1).

Observou-se que a situação ocupacional da mãe, tabagismo e infecções maternas no período pré-natal, depressão referida na gestação e indicadores maternos de transtorno mental comum, quando a criança tinha 1 ano, apresentaram uma associação estatística significativa com o risco para o desenvolvimento (Tabelas 2 e 3 ).

O TMC materno associou-se significativamente, também, com risco para o desenvolvimento das subáreas: motor fino e linguagem (Tabela 4). 
Tabela 1. Desempenho global das crianças, no teste neuropsicomotor do Denver II e nas 4 áreas especificas (linguagem, pessoal social, motor fina e motor ampla).

\begin{tabular}{lc}
\hline Desempenho no Teste DENVER II & $\mathbf{f}(\%)$ \\
\hline Classificação Global & $37(56,9)$ \\
Normal & $28(43,1)$ \\
Risco & \\
Área Específica & \\
Linguagem & $39(60,0)$ \\
$\quad$ Normal & $10(15,4)$ \\
Cuidados & $16(24,6)$ \\
Atrasos & \\
Pessoal-Social & $56(55,4)$ \\
Normal & $25(38,5)$ \\
Cuidados & $4(6,10)$ \\
Atrasos & \\
Motora Fina & $52(80,0)$ \\
Normal & $0,0(0,0)$ \\
Cuidados & $13(20,0)$ \\
Atrasos & \\
Motora ampla & $65(100,0)$ \\
Normal & $0,0(0,0)$ \\
Cuidados & $0,0(0,0)$ \\
Atrasos &
\end{tabular}

Frequência (f) e Porcentagem (\%).

Ao se incluir as variáveis que apresentaram diferenças significativas no modelo de regressão logística multivariada, observou-se que o transtorno mental comum (TMC) materno manteve-se como fator de risco, ou seja, pontuação indicativa de TMC aumentou a probabilidade de risco para o desenvolvimento infantil $(\mathrm{OR}=13,8)$. Por outro lado, a mãe trabalhar fora se constituiu em fator de proteção, reduzindo a probabilidade de risco para o desenvolvimento da criança $(\mathrm{OR}=0,095)$ (Tabela 5).

\section{Discussão}

A porcentagem de crianças que apresentaram atraso de desenvolvimento neste estudo $(43,1 \%)$, com maiores prejuízos na linguagem, está acima dos índices de risco para o desenvolvimento encontrado em outras pesquisas realizadas no contexto brasileiro, com o mesmo instrumento de rastreamento de risco para o desenvolvimento. A organização Mundial de Saúde (OMS) estima que $10 \%$ da população de qualquer país seja constituída por pessoas com algum tipo de deficiência $^{1}$. Santa Maria-Mengel ${ }^{27}$ observou que 33\% das crianças cadastradas em um Programa de Saúde da Família, em Ribeirão Preto (SP), apresentavam risco para o desenvolvimento, em sua maioria com atrasos na área da linguagem. Pilz e Scherman encontraram prevalência de suspeita de atraso no desenvolvimento neuropsicomotor de $27 \%$ em uma amostra de crianças de até 6 anos residentes no município de Canoas (RS) ${ }^{30}$. Halpern et al. ${ }^{2}$ verificaram que, aos 12 meses, 34\% das crianças nascidas em Pelotas (RS), em 1993, apresentavam suspeita de atraso no desenvolvimento. É preciso considerar que Santa MariaMenguel $^{27}$ e Pilz e Scherman ${ }^{30}$ pesquisaram uma faixa etária com maior amplitude, em que as crianças tinham de 6 a 44 meses de idade e 0 a 6 anos, respectivamente, e, no estudo de Halpern et al. $^{2}$, a amostra aleatória provinha de uma coorte de crianças nascidas nos hospitais de Pelotas, RS em 1993, sem selecionar as que viviam em bairros periféricos, com condições socioeconômicas desfavoráveis, como no presente estudo. Mas muito possivelmente, a hipótese mais plausível para explicar os resultados esteja associada à alta porcentagem de transtornos mentais comuns detectados nas mães, que aumentaram em $13,8 \%$ o risco para atraso no desenvolvimento e serão discutidos mais adiante.

Antes de analisar os altos índices de TMC materno, é preciso ressaltar que em relação ao atraso no desenvolvimento, vários dos fatores apontados pela literatura como de risco não estavam presentes nesta amostra de sujeitos. Todas as crianças eram nascidas a termo, em sua maioria por parto normal, com peso por volta de 3.120 grs e $92 \%$ tiveram alta junto com a mãe. As que não tiveram alta conjunta permaneceram no hospital para fazer banho de luz. Esses são índices mais favoráveis que os da cidade em que se realizou o estudo e do Brasil ${ }^{31}$.

Durante o primeiro ano de vida, $89,2 \%$ das crianças foi amamentada no peito, até aproximadamente oito meses, que, segundo Halpern et al. ${ }^{2}$, pode contribuir para menor risco de atraso e melhor desempenho no desenvolvimento cognitivo futuro. O tempo médio de desmame das crianças não foi baixo, considerando que se preconiza amamentação elusiva no peito até os 6 meses de vida. A maioria estava com a vacinação em dia, e as mães referiram que tinham sono normal e alimentavam-se bem. Em relação aos marcos do desenvolvimento (sustentar a cabeça, sentar, engatinhar e andar), a grande maioria adquiriu essas habilidades dentro do período esperado e $40 \%$ da amostra já andava no início do primeiro ano de vida. Desta forma, pode-se 
Tabela 2. Associação entre variáveis maternas e os indicadores de desenvolvimento global da criança.

\begin{tabular}{|c|c|c|c|}
\hline \multirow{2}{*}{ Variáveis } & \multicolumn{2}{|c|}{ DENVER GLOBAL } & \multirow{2}{*}{$\mathrm{p}$} \\
\hline & Normal - f (\%) & Risco - f (\%) & \\
\hline \multicolumn{4}{|l|}{ Idade materna } \\
\hline$<$ de 20 anos & $6(35,3)$ & $11(64,7)$ & \multirow{3}{*}{0,09} \\
\hline 20 a 30 anos & $21(67,7)$ & $10(32,3)$ & \\
\hline 31 ou mais & $10(58,8)$ & $7(41,2)$ & \\
\hline \multicolumn{4}{|l|}{ Escolaridade materna } \\
\hline Até 3 & $7(58,3)$ & $5(41,7)$ & \multirow{3}{*}{0,98} \\
\hline De 4 a 7 & $7(58,3)$ & $5(41,7)$ & \\
\hline 8 ou mais & $23(56,1)$ & $18(43,9)$ & \\
\hline \multicolumn{4}{|l|}{ Situação Ocupacional Materna } \\
\hline Desempregada & $26(50,0)$ & $26(50,0)$ & \multirow[t]{2}{*}{$0,03^{\star}$} \\
\hline Empregada & $11(84,6)$ & $2(15,4)$ & \\
\hline \multicolumn{4}{|l|}{ Ocupação Materna } \\
\hline Não Qualificada & $7,0(77,8)$ & $3,0(22,2)$ & \multirow[t]{2}{*}{1,00} \\
\hline Qualificada & $3,0(100)$ & $0,0(0,0)$ & \\
\hline \multicolumn{4}{|l|}{ Marido/companheiro } \\
\hline Não & $2,0(66,7)$ & $1,0(33,3)$ & \multirow[t]{2}{*}{0,60} \\
\hline Sim & $35(56,5)$ & $27(43,5)$ & \\
\hline \multicolumn{4}{|c|}{ Tabagismo e infecções pré-natais } \\
\hline Não & $24(68,6)$ & $11(31,4)$ & \multirow[t]{2}{*}{$0,04^{\star}$} \\
\hline Sim & $13(43,3)$ & $17(56,7)$ & \\
\hline \multicolumn{4}{|l|}{ Depressão na gestação } \\
\hline Ausência & $34(63,0)$ & $20(37,0)$ & \multirow[t]{2}{*}{$0,04^{\times}$} \\
\hline Presença & $3(24,3)$ & $8(72,7)$ & \\
\hline \multicolumn{4}{|l|}{ Transtorno Mental Comum } \\
\hline Não & $28(77,8)$ & $8(22,2)$ & 0 \\
\hline $\operatorname{Sim}$ & $9(31,0)$ & $20(69,0)$ & $001^{\star}$ \\
\hline
\end{tabular}

Frequência (f) e Porcentagem (\%) Mediana (Med) Mínimo (Min) Máximo (Max) Qui-Quadrado e Exato de Fisher * p $<0,05$.

supor que se tratava de um grupo de crianças saudáveis.

Apesar da baixa renda familiar (Méd. 1,64 salários mínimos) e das moradias estarem localizadas em bairros periféricos, as condições habitacionais não eram de todo desfavoráveis. A grande maioria morava em casas com rede de água, esgoto, luz e asfalto, com baixa densidade habitacional. Com relação à constituição familiar, a maior parte dos pais era jovem e tinha 8 ou mais anos de estudo. Apesar de a minoria exercer ocupação qualificada somente três pais estavam desempregados. A grande maioria das mães morava com o companheiro e metade exercia trabalho remunerado.

A mãe ter um emprego se mostrou fator de proteção para o desenvolvimento infantil. Os dados da literatura ainda não são conclusivos quanto ao papel da ocupação materna no desenvolvimento da criança ${ }^{7,32,33}$. Por um lado, verificou-se que as mães que trabalhavam tinham menor disponibilidade para atividades promotoras de desenvolvimento cognitivo, menos tempo em leitura com seus filhos, e esse tempo não era compensado pelo pai ${ }^{32}$. Especificamente com crianças menores, o emprego materno em tempo integral, no primeiro ano de vida da criança, mostrou efeito negativo sobre o desenvolvimento cognitivo, mas não foi uniforme e esteve condicionado a diferentes tipos de família ${ }^{33}$.

Fazendo contraponto com estes achados, há pesquisas que identificaram o trabalho materno como fator de proteção. Por ser elemento gerador de renda, o trabalho materno facilitaria o acesso a brinquedos e outros recursos possibilitando, indiretamente, uma maior estimulação da criança, com efeitos na sua cognição. Ainda, a satisfação ocupacional da mãe promovia sua autoestima, motivando experiências positivas na relação com seus filhos ${ }^{7,34}$. Sugere-se que em próximos estudos essa variável receba atenção especial, detalhando melhor a idade da criança quan- 
Tabela 3. Associação entre variáveis paternas, socioeconômicas e demográficas e os indicadores de desenvolvimento global da criança.

\begin{tabular}{|c|c|c|c|}
\hline \multirow{2}{*}{ Variáveis } & \multicolumn{2}{|c|}{ DENVER GLOBAL } & \multirow{2}{*}{$\mathrm{p}$} \\
\hline & Normal - f (\%) & Risco - f (\%) & \\
\hline \multicolumn{4}{|l|}{ Escolaridade Paterna } \\
\hline Até 3 anos & $5(45,5)$ & $6(54,5)$ & \multirow{3}{*}{0,68} \\
\hline De 4 a 7 anos & $8(57,1)$ & $6(42,9)$ & \\
\hline 8 ou mais anos & $24(60,0)$ & $16(40,0)$ & \\
\hline \multicolumn{4}{|l|}{ Idade Paterna } \\
\hline$<$ de 20 anos & $2(66,7)$ & $1(33,3)$ & \multirow{3}{*}{0,83} \\
\hline 20 a 30 anos & $21(53,8)$ & $18(46,2)$ & \\
\hline 31 ou mais & $14(60,9)$ & $9(39,1)$ & \\
\hline \multicolumn{4}{|c|}{ Situação Ocupacional Paterna } \\
\hline Desempregado & $6(85,7)$ & $1(14,3)$ & \multirow[t]{2}{*}{0,13} \\
\hline Empregado & $31(53,4)$ & $27(46,6)$ & \\
\hline \multicolumn{4}{|l|}{ Ocupação Paterna } \\
\hline Não Qualificada & $18(52,9)$ & $16(47,1)$ & \multirow[t]{3}{*}{1,00} \\
\hline \multirow[t]{2}{*}{ Qualificada } & $13(54,2)$ & $11(45,8)$ & \\
\hline & Med (min- max) & Med (min- max) & \\
\hline \multicolumn{4}{|l|}{ Renda per capta } \\
\hline Em salários & $1,72(0,21-4,3)$ & $1,43(0,64-3,87)$ & 0,23 \\
\hline \multicolumn{4}{|l|}{ Número de pessoas na casa } \\
\hline Em número & $4(3-13)$ & $5(3-9)$ & 0,55 \\
\hline
\end{tabular}

Frequência (f) e Porcentagem (\%) Qui-Quadrado * $\mathrm{p}<0,05$

Tabela 4. Relação entre transtorno mental da mãe e atrasos no desenvolvimento dos domínios pessoal social, motor fina e linguagem.

\begin{tabular}{|c|c|c|c|c|}
\hline \multirow{2}{*}{ TMC } & \multicolumn{3}{|c|}{ Área Específica DENVER II } & \multirow{2}{*}{$\mathbf{p}$} \\
\hline & Normal f (\%) & Cuidado f (\%) & Atraso f $(\%)$ & \\
\hline \multicolumn{5}{|c|}{ PESSOAL SOCIAL } \\
\hline Não & $23(63,9)$ & $11(30,6)$ & $2(5,6)$ & \multirow[t]{2}{*}{0,29} \\
\hline Sim & $13(44,8)$ & $14(48,3)$ & $2(6,9)$ & \\
\hline \multicolumn{5}{|c|}{ MOTOR FINO } \\
\hline Não & $32(88,9)$ & $0(0)$ & $4(11,1)$ & \multirow[t]{2}{*}{$0,04^{*}$} \\
\hline Sim & $20(69,0)$ & $0(0)$ & $9(31,0)$ & \\
\hline \multicolumn{5}{|c|}{ LINGUAGEM } \\
\hline Não & $26(72,2)$ & $5(13,9)$ & $5(13,9)$ & \multirow[t]{2}{*}{$0,02^{*}$} \\
\hline Sim & $12(41,4)$ & $5(17,2)$ & $12(41,4)$ & \\
\hline
\end{tabular}

Frequência (f) e Porcentagem (\%) Qui-Quadrado * $\mathrm{p}<0,05$

Tabela 5. Modelo de regressão logística.

\begin{tabular}{lrrr}
\hline \multicolumn{1}{c}{ Variáveis } & \multicolumn{1}{c}{$\boldsymbol{p}^{*}$} & \multicolumn{1}{c}{$\boldsymbol{O R}$} & \multicolumn{1}{c}{$\boldsymbol{I C}(\mathbf{9 5 \% )}$} \\
\hline TMC & 0,000 & 13,8 & $(3,09-62,25)$ \\
Mãe trabalhar fora & 0,02 & 0,095 & $(0,01-0,72)$ \\
Depressão na gestação & 0,85 & 1,19 & $(0,18-7,64)$ \\
Risco pré- natal & 0,10 & 0,34 & $(0,09-1,24)$
\end{tabular}

Odds Ratio (OR) Intervalo de confiança (IC) * $\mathrm{p}<0,05$ do do ingresso da mãe no mercado de trabalho, o número de horas que a mãe permanece fora, a flexibilidade quanto ao seu cumprimento, para poder avaliar com mais precisão seu papel no desenvolvimento de crianças desta faixa etária.

Todas as mães relataram ter feito acompanhamento do pré-natal em suas respectivas unidades de saúde. O comparecimento às consultas 
de pré-natal permite o diagnóstico e o tratamento de complicações durante a gestação e a consequente redução ou eliminação de riscos passíveis de serem corrigidos, como as síndromes hipertensivas, sífilis congênita e infecções urinárias complicadas $^{35}$. Apesar do acompanhamento médico durante a gestação, várias continuaram fumando, tiveram infecções, principalmente urinárias e 17\% referiram depressão. Essas intercorrências mostraram associação significativa com risco para o desenvolvimento.

A porcentagem de mães que referiram depressão durante a gestação foi similar à encontrada em outros trabalhos ${ }^{11}$. A gestação e o puerpério são os períodos da vida da mulher com maior prevalência de transtornos mentais, principalmente no primeiro e no terceiro trimestre de gestação e nos primeiros 30 dias de puerpério, quando ocorrem inúmeras alterações físicas, hormonais, psíquicas e de inserção social, que podem se refletir diretamente na saúde mental materna. Em geral, a depressão gestacional não se constitui um episódio único, mas observamse elevadas taxas de reincidência na evolução do quadro e é um forte fator de risco para depressão e outros transtornos mentais na fase pósnatal $^{11,12}$. Apesar da depressão gestacional não ter sido avaliada a partir de instrumento padronizado, mas apenas referida pelas mães, ela poderia explicar, em parte, o alto índice de transtorno mental comum (TMC) observado na avaliação posterior.

Foi alta a porcentagem de mães que pontuou para TMC (44,6\%), quando comparada a outras pesquisas desenvolvidas em Unidades de Saúde ${ }^{21-24}$ possivelmente por se tratar de amostra composta exclusivamente por mulheres, moradoras de áreas pobres da cidade. Diversos trabalhos vêm apontando para maiores índices de TMC em mulheres que nos homens. Maragno et al. ${ }^{23}$ pesquisando a prevalência de TMC em moradores de áreas periféricas do estado de São Paulo cobertas pela ESF, encontraram uma prevalência significativamente maior nas mulheres, idosos e nas categorias de menor renda ou de menor escolaridade. Lima et al. ${ }^{22}$, desenvolvendo um estudo transversal populacional no mesmo município em que foi realizada a atual pesquisa, também mostraram associação entre os TMC e os indicadores de desvantagem social e sexo.

Apesar de algumas características desta amostra, como sexo e local de moradia, serem fatores de risco para TMC, outros como idade materna, família constituída, grupo de apoio, escolaridade e renda média não parecem justificar os altos índices de TMC encontrados. Possivelmente, outras condições não investigadas na pesquisa como frustrações, conflitos conjugais, violência doméstica e mesmo acontecimentos diários menores podem ter influenciado os resultados. Com os dados disponíveis, é possível levantar a hipótese de que essas mulheres estavam vivenciando uma mudança na sua rotina, que além de se ocupar dos afazeres domésticos, tinham que cuidar de filhos pequenos, muitas vezes vistos como birrentos e nervosos, nem sempre uma tarefa prazerosa, com consequências para sua saúde mental. A alta sobrecarga doméstica e ter filhos foram apontados como possíveis fatores de risco para transtorno mental em mulheres ${ }^{21}$.

Na regressão logística, os indicadores de TMC foram preditivos de risco para o desenvolvimento global, aumentando em 13,8 vezes a chance de atraso da criança. Não se encontraram, na literatura, pesquisas que relacionassem TMC materno e desenvolvimento infantil. Na medida em que o conceito de TMC abrange manifestações de sofrimento psíquico, sem necessariamente preencher todos os critérios diagnósticos para transtornos depressivos, ansiosos e somatoformes ${ }^{24}$, os dados serão discutidos a partir de trabalhos que pesquisaram a relação entre sintomas de depressão e ansiedade materna e desenvolvimento infantil. Há pesquisas mostrando que sintomas psiquiátricos leves ou mesmo quadros em nível subclínico podem associar-se com altos níveis de problemas comportamentais e de temperamento, como desatenção e falta de regulação da emoção ${ }^{36}$ e podem contribuir, também, para déficits cognitivos e de linguagem ${ }^{12}$.

Especificamente com relação à depressão pósparto, são muitos os autores que observaram que ela pode afetar a disponibilidade cognitiva e emocional da mãe, que passa a apresentar dificuldade de se conectar às necessidades reais do bebê, sendo menos responsiva, com prejuízos para o desenvolvimento infantil ${ }^{14,15}$. Ela própria se percebe menos competente para cuidar adequadamente de seu filho e exercer seu papel de mediadora frente ao desenvolvimento da criança ${ }^{10}$.

Motta et al. ${ }^{14}$ em levantamento dos trabalhos experimentais sobre a relação entre a depressão pós-parto e o desenvolvimento da criança, observaram que as mães eram mais intrusivas, menos sensíveis e empáticas, apáticas na interação, com pouca disponibilidade para brincar, e, como as mães desta pesquisa, descreviam a criança de forma negativa. As crianças, por sua vez, tinham menos envolvimento com suas mães, menos comportamentos exploratórios, irritabilidade, apego 
inseguro e atraso de desenvolvimento neuropsicomotor, com baixos índices de linguagem expressiva.

Assim como ocorreu na presente avaliação, a área que apresentou maiores prejuízos foi a linguagem, possivelmente porque mães com comprometimento psiquiátrico estão menos disponíveis para a interação social, e se comunicam menos com seus filhos ${ }^{15}$.

A depressão materna não afeta apenas o desenvolvimento inicial, mas pode influir a médio e longo prazo, pois mulheres com história de depressão na gestação e pós-parto são mais vulneráveis a novos episódios depressivos ou apresentam quadros depressivos persistentes ${ }^{12}$. Cornish et al. ${ }^{37}$ observaram que crianças de 12 meses apresentavam taxas elevadas de atraso no desenvolvimento relacionadas à depressão materna crônica. Dependendo da data de início e do tempo de exposição à depressão materna, houve diferença na gravidade dos problemas comportamentais apresentados por crianças em idade préescolar ${ }^{15}$. Outros estudos relataram o impacto negativo da cronicidade dos sintomas depressivos maternos em crianças pré-escolares, quando se verificou que elas eram mais vulneráveis a apresentar prejuízos no desenvolvimento cognitivo e da linguagem ${ }^{12}$.

Como a presente pesquisa foi um estudo transversal e as mães só foram avaliadas quando a criança tinha 1 ano, não é possível concluir que o alto índice de risco para o desenvolvimento apresentado pelas crianças, especialmente na área da linguagem, fosse devido à cronicidade dos transtornos maternos. Entretanto, mesmo que seja um episódio esporádico de sofrimento psíquico, ele está ocorrendo em um momento de grandes mudanças nas habilidades da criança se comunicar, a segunda metade do primeiro ano de vida, e depende de uma adequada mediação materna. É a fase em que ela começa a prestar atenção na mímica e a procurar ativamente os objetos nomeados pela mãe, compartilha a exploração dos objetos com o adulto, e se espera que a mãe tenha a habilidade de coordenar as perspectivas e as ações da criança, dê respostas contingentes para possibilitar um desenvolvimento adequado da cognição e da linguagem ${ }^{12}$. Os riscos que os quadros mentais crônicos e o momento em que a criança foi exposta a eles podem acarretar para o desenvolvimento infantil, sugere-se que em próximos trabalhos essa variável seja melhor investigada, em estudos com delineamento longitudinal.

Em relação ao papel da ansiedade materna no desenvolvimento, por um lado, se alguns estudos com mães de bebês nascidos a termo, a ansiedade associou-se a efeitos prejudiciais ao desenvolvimento da criança a curto e médio pra$\mathrm{zo}^{18}$, por outro lado, um estudo com mães de crianças nascidas pré-termo, mostrou que a ansiedade materna teve um efeito diferencial nas subáreas do desenvolvimento. A ansiedade materna atuou como mecanismo de proteção ao desenvolvimento cognitivo e como fator de risco ao desenvolvimento motor $\mathrm{amplo}^{38}$. O presente estudo não reforça esses dados na medida em que todas as crianças tiveram um desenvolvimento motor amplo normal, incluindo as crianças cujas mães pontuaram para TMC.

\section{Considerações finais}

A constatação de um alto índice de possíveis riscos ao desenvolvimento em crianças de 1 ano, sem risco biológico, moradores em bairros periféricos, vem reforçar a necessidade de implementar, na atenção primária, práticas para avaliar a condição de desenvolvimento das crianças, dentro de uma visão integrada da saúde infantil, como proposto pela OPAS, através da estratégia de Atenção Integrada às Doenças Prevalentes (AIDPI). O acompanhamento do desenvolvimento deve ocorrer, especialmente, nos dois primeiros anos de vida, etapa extrauterina em que o tecido nervoso mais se desenvolve e está mais sujeito a agravos. Devido à plasticidade, é também nessa época, que a criança melhor responde a terapias e aos estímulos que recebe do ambiente. O impacto positivo que os programas de intervenção precoce têm mostrado em relação ao desenvolvimento da criança e seu posterior desempenho escolar justifica de forma definitiva a necessidade da identificação precoce das crianças com risco de atraso ${ }^{8}$.

No entanto, o alto índice de mães com transtorno mental comum, preditor de risco para o desenvolvimento infantil, reforça a necessidade dos serviços de saúde levarem em conta o binômio mãe/filho, isto é, ao lado de estratégias e orientações preocupadas com o desenvolvimento infantil, implementar ações que tenham por objetivo preservar e cuidar da saúde mental materna. 


\section{Colaboradores}

DG Ribeiro, GB Perosa e FHP Padovani participaram igualmente de todas as etapas de elaboração do artigo.

\section{Agradecimentos}

À CAPES (Coordenação de Aperfeiçoamento de Pessoal de Nível Superior).

\section{Referências}

1. Figueiras ACM, Puccini RF, Silva EMK, Pedromônico MRM. Avaliação das práticas e conhecimentos de profissionais da atenção primária à saúde sobre vigilância do desenvolvimento infantil. Cad Saude Publica 2003; 19(6):1691-1699.

2. Halpern R, Giugliani ERJ, Victora CG, Barros FC, Horta BL. Fatores de risco para suspeita de atraso no desenvolvimento neuropsicomotor aos $12 \mathrm{me}$ ses de vida. J Pediatr 2000; 76(6):421-428.

3. Rutter M, Sroufe LA. Developmental psychopathology: concepts and challenges. Dev Psychopatol 2000; 12(3):265-296.

4. Bradley H, Corwyn RF. Socioeconomic status and child development. Annu Rev Psychol 2002; 53:371399.

5. Lamy Filho F, Medeiros S M, Lamy Z C, Moreira M E L. Ambiente domiciliar e alterações do desenvolvimento em crianças de comunidade da periferia de São Luís - MA. Cien Saude Colet 2011; 16(10):41814187.

6. Lordelo ER, Fonseca AL, Araújo MLVB. Responsividade do ambiente e desenvolvimento: crenças e práticas como sistema cultural de criação de filhos. Psicol Reflex Crit 2000; 13(1):1-8.

7. Andrade SA, Santos DN, Bastos AC, Pedromônico MRM, Almeida-Filho N, Barreto ML. Ambiente familiar e desenvolvimento cognitivo infantil: uma abordagem epidemiológica. Rev Saude Publica 2005; 39(4):606-611.

8. Figueiras ACM, Souza ICN, Rios VG, Benguigui Y. Manual para vigilância do desenvolvimento infantil no contexto da AIDPI: módulo II. Washington: OPAS; 2005.

9. Lautch MM, Esser G, Schmidt MH. Differential development of infants at risk for psychopathology: the moderating role of early maternal responsive. Dev Med Child Neurol 2001; 43(5):292-300.

10. Frizzo GB, Piccinini CA. Interação mãe-bebê em contexto de depressão materna: aspectos teóricos e empíricos. Psicol Estud 2005; 10(1):47-55.

11. Pereira PK, Lovisi GM. Prevalência da depressão gestacional e fatores associados. Rev Psiquiatr Clín 2008; 35(4):144-153.

12. Sohr-Preston SL, Scaramella LV. Implications of timing of maternal depressive symptoms for early cognitive and language development. Clin Child Fam Psychol Rev 2006; 9(1):65-78.

13. Schwengber DD, Piccinini CA. A experiência da maternidade no contexto da depressão materna no final do primeiro ano de vida do bebê. Estud. Psicol 2005; 22(2):143-156.

14. Motta MG, Lucion AB, Manfro GG. Efeitos da depressão materna no desenvolvimento neurobiológico e psicológico da criança. Rev Psiquiatr 2005; 27(2):165-176.

15. Grace SL, Evindar A, Stewart DE. The effect of postpartum depression on child cognitive development and behavior: a review and critical analysis of the literature. Arch Womens Ment Health 2003; 6(4):263274.

16. Faisal-Cury A, Menezes P. Depressão durante a gravidez no sistema público de saúde brasileiro. Rev Bras Psiquiatr 2010; 32(2):464-465. 
17. Glasheen C, Gale A, Fabio A. A systematic review of the effects of postnatal maternal anxiety on children. Arch Womens Ment Health 2010; 13(1):61-74.

18. Clavarino A, Mamun AA, O'Callaghan M, Aird R, Bor W, O'Callaghan F, Williams GM, Marrington S, Najman JM, Alati R. Maternal anxiety and attention problems in children at 5 and 14 years. $J$ Atten Dis 2010; 13(6):658-667.

19. Engle PL. Maternal mental health: program and policy implications. J Clin Nutr 2009; 89(3):963970.

20. Zanardo V, Gasparetto S, Giustardi A, Suppiej A, Trevisanuto D, Pascoli I, Freato F. Impact of anxiety in the puerperium on breast-feeding outcomes: role of parity. J Pediatr Gasttroenterol Nutr 2009; 49(5):631-634.

21. Araújo TM, Pinho OS, Almeida MMG. Prevalência de transtornos mentais comuns em mulheres e sua relação com as características sociodemográficas e o trabalho doméstico. Rev Bras Saúde Mater Infant 2005; 5(3):337-348.

22. Lima MCP, Menezes PR, Carandina L, Cesar CLG, Barros MBA, Goldbaum M. Common mental disorders and the use of psychoactive drugs: the impact of socioeconomic conditions. Rev Saude Publica 2008; 42(4):717-723.

23. Maragno L, Goldbaum M, Gianini RJ, Novaes HMD, Cesar CL. Prevalência de Transtorno mental comum em populacões atendidas pelo Programa Saúda da família (QUALIS) no município de São Paulo, Brasil. Cad Saude Publica 2006; 22(8):1639-1648.

24. Fonseca MLG, Guimarães MBL, Vasconcelos, EM. Sofrimento difuso e transtornos mentais comuns: uma revisão bibliográfica. Rev. APS 2008; 11(3):285294.

25. Frankenburg WK, Dodds JB. Denver II: training manual. Denver: Denver Developmental Materials; 1999.

26. Figueiras A, Pedromônico M, Sales L, Figueiras S. Manual para vigilância do desenvolvimento da criança de 0 a 2 anos de idade na atenção primária à saúde. Belém: Secretaria Municipal de Saúde de Belém; 2000.

27. Santa-Maria-Mengel MRS. Vigilância do desenvolvimento em Programa de Saúde da Família: triagem para detecção de riscos para problemas de desenvolvimento em crianças [tese]. Ribeirão Preto: Faculdade de Medicina de Ribeirão Preto; 2007.

28. Mari JJ, Williams P. A validity study of a Psychiatric Screening Questionnaire (SRQ-20) in primary care in the city of São Paulo. Br J Psychiatry 1986; 148:2326.
29. Gonçalves DM, Stein AT, Kapczinski F. Avaliação de desempenho do Self-Reporting Questionnaire como instrumento de rastreamento psiquiátrico: um estudo comparativo com o Structured Clinical Interview for DSM-IV-TR. Cad Saude Publica 2008; 24(2):380-390.

30. Pilz E M L, Schermann L B. Determinantes biológicos e ambientais no desenvolvimento neuropsicomotor em uma amostra de crianças de Canoas/ RS. Cien Saude Colet 2007; 12(1):181-190.

31. IBGE. Indicadores sociodemográficos e de saúde no Brasil. Rio de Janeiro: IBGE; 2008.

32. Cawley J, Liu F. Mechanisms for the association between maternal employment and child cognitive development. NBER Working Paper 2007; 13609:125.

33. Hill JL, Waldfogel J, Brooks-Gunn J, Han WJ. Maternal employment and child development: a fresh look using newer methods. Dev Psychol 2005; 41(6):833-850.

34. Bianchi SM. Maternal employment and time with children's: dramatic change or surprise continuity? Demography 2000; 37(4):401-414.

35. Kilsztajn S, Rossbach AC, Carmo MSN, Sugahara GTL. Assistência pré-natal, baixo peso e prematuridade no Estado de São Paulo, 2000. Rev Saude Publica. 2003; 37(3):303-310.

36. West AE, Newman DL. Worried and blue: mild parental anxiety and depression in relation to the development of young children's temperament and behavior problems. Parent Sci Pract 2003; 3(2):133-

37. 154 .

Cornish AM, McMahon CA, Ungerer JA, Barnett B, Kowalenko N, Tennant C. Postnatal depression and infant cognitive and motor development in the second postnatal year: the impact of depression chronicity and infant gender. Infant Behav Dev 2005;

38. 28(4):407-417.

Fraga DA, Linhares, MBM, Carvalho AE, Martinez FE. Desenvolvimento de bebês prematuros relacionado a variáveis neonatais e maternas. Psicol Estud 2008; 13(2):335-344.

Artigo apresentado em 16/10/2012

Aprovado em 15/01/2013

Versão final apresentada em 22/01/2013 